International Journal of Current Advanced Research

ISSN: O: 2319-6475, ISSN: P: 2319 - 6505, Impact Factor: SJIF: 5.995

Available Online at www.journalijcar.org

Volume 6; Issue 3; March 2017; Page No. 2480-2487

DOI: http://dx.doi.org/10.24327/ijcar.2017.2487.0033

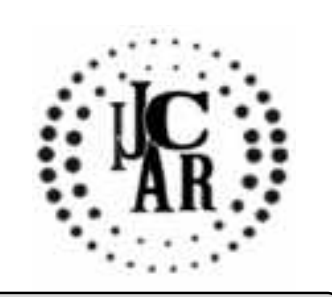

Research Article

\title{
DETERMINATION OF THERMODYNAMIC STABILITY CONSTANT OF BIVALENT TRANSITION METAL COMPLEXES WITH SCHIFFS BASE AS LIGANDS
}

\author{
Prem Mohan Mishra ${ }^{1}$, Sanjay Kumar Mishra ${ }^{2}$., Sri Jai Prakash Mishra ${ }^{3}$ and Veena Mishra ${ }^{4}$ \\ ${ }^{1}$ Dept. of Chemistry M L S M College, Darbhanga, Bihar-846004 \\ 2Vill - Kahara, Dist - Saharsa \\ ${ }^{3}$ Dighiwest, Darbhanga- 846004
}

\section{A R T I C L E I N F O}

Article History:

Received $15^{\text {th }}$ December, 2016

Received in revised form $19^{\text {th }}$ January, 2017

Accepted $12^{\text {th }}$ February, 2017

Published online $28^{\text {th }}$ March, 2017

\begin{abstract}
A B S T R A C T
In continuation of our previous work here also, we have been interested in studying the stability of complexes of transition metals with biologically active ligands.

Here we are presenting determination of stability constant of complex compounds using ligands synthesised from 5-Methyl furan-2-carbaldehyde and 7- methoxy naphthalene- 2 amine with bivalent transition metals i.e. $\mathrm{Co}(\mathrm{II}), \mathrm{Ni}(\mathrm{II}), \mathrm{Cu}(\mathrm{II})$ and $\mathrm{Zn}(\mathrm{II})$.

Schiff's base ligands were synthesized by the condensation reaction of 5-Methyl furan-2carbaldehyde with 7- Methoxy naphthalene - 2 - amine. Nitrate salts of divalent cobalt, nickel, copper and zinc were estimated by standard volumetric and gravimetric methods. Ligand was analysed for elements by reported methods. $\mathrm{pH}$ metric titrations were carried out in an inert atmosphere of nitrogen gas at constant ionic strength of $0.1 \mathrm{M} \mathrm{KNO}_{3}$ with the help of digital $\mathrm{pH}$ meter.Stability constant of complexes of these metals with the ligands synthesized were computed by Irving- Rossoti technique ${ }^{1}$ modified by Calvin Bjerrum ${ }^{2}$ at temperatures $298 \mathrm{~K}$.

The stability constant values of metals for the given ligand at the given temperature were found to be in the order $\mathrm{Cu}$ (II) $>\mathrm{Ni}$ (II), $\mathrm{Co}$ (II) $>\mathrm{Zn}$ (II).

This result is in agreement with the natural order proposed by Irving-William.
\end{abstract}

Copyright $@ 2017$ Prem Mohan Mishra et al. This is an open access article distributed under the Creative Commons Attribution License, which permits unrestricted use, distribution, and reproduction in any medium, provided the original work is properly cited.

\section{INTRODUCTION}

Schiff's base offers a versatile and flexible series of ligands capable to bind with variety of metal ions to give complexes with varying properties. These complexes are biologically active $^{3}$, have wide potential applications in many fields such as catalysis, ${ }^{4}$ electrochemistry ${ }^{5}$ and medicines like anti tumour, anti viral ${ }^{6}$, anti cancer ${ }^{7}$ and other many anti bacterial agents ${ }^{8}$. Metal ions play vital role in a vast number of widely different biological processes. The interaction of these ions with biologically active ligands is a subject of considerable interest. Some of the biologically active compounds act via chelation $^{8}$. A large number of such chelates have been synthesized, their structure and activity have been studied, but little is known about its stability in solution which will be its functioning condition.

\section{Experimental}

Nitrate salts of divalent $\mathrm{Co}, \mathrm{Ni}, \mathrm{Cu}$ and $\mathrm{Zn}$ all were E. Merck. All other chemicals used were Anal R grade and used without further purification. Elemental analysis of metal salts were done by volumetric and gravimetric methods ${ }^{11}$.

\section{*Corresponding author: Prem Mohan Mishra}

Dept. of Chemistry M L S M College, Darbhanga, Bihar-846004 medium in the ratio $3: 2(\mathrm{v} / \mathrm{v})$. Dioxane was purified by standard method.

Schiff's base ligands were synthesised by reported method ${ }^{12}$ $1.5 \mathrm{~g}$ of 3-Methyl furan-2-carbaldehyde in solution were mixed with $3.5 \mathrm{~g} 7$ - Hydroxy naphthalene -2 - amine. The mixture was refluxed in the presence of glacial acetic acid for 3 hours. The solution was concentrated and cooled to $0^{0} \mathrm{C}$. The product obtained was filtered, washed several times and recrystallised from ethanol. The yield of product was nearly $2.40 \mathrm{~g}$

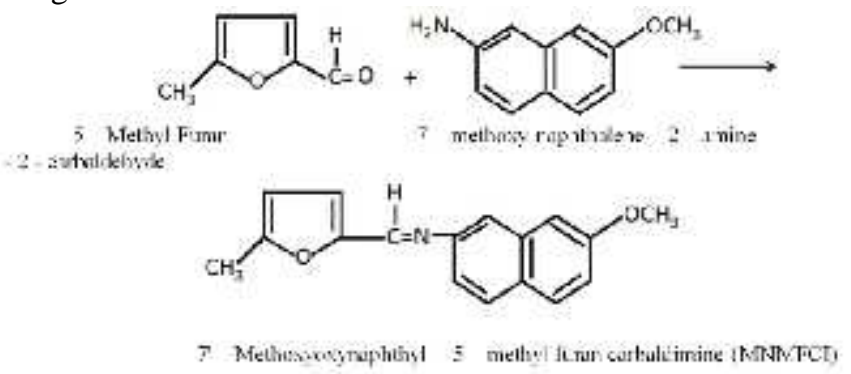

Solution of metal salts, ligands, electrolytes etc were prepared in doubly distilled $\mathrm{CO}_{2}$ free water. Strength of various solutions used were tabulated in Table -1 . 
$\mathrm{pH}$ metric titration of acid, acid + ligand and acid + ligand + metal ion solutions were done at constant ionic strength of 0.1 $\mathrm{M} \mathrm{KNO}_{3}$ at temperature $298 \mathrm{~K}$ in an inert atmosphere of nitrogen.

The same process of titration were repeated for all the four $\mathrm{Co}, \mathrm{Ni}, \mathrm{Cu}$ and $\mathrm{Zn}$ metal ions. The change in colour and appearance of turbidity at particular $\mathrm{pH}$ value were recorded simultaneously.

The change in $\mathrm{pH}$ of the solutions with each addition of alkali was recorded in Table No. 2.

A graph was plotted between $\mathrm{pH}$ meter reading [B] and volume of alkali added in each case. Graph no. 1. The three titration curves so obtained for each metal ions are referred as:

1. Acid titration curve (a)

2. Ligand titration curve (b)

3. Complex titration curve (c) respectively

\section{Calculation OF $\bar{n}_{A}, \bar{n}$ AND $\mathbf{P}^{\mathbf{L}}$}

The $\bar{n}_{A}, \bar{n} \& \mathrm{P}^{\mathrm{L}}$ are calculated using standard expressions

$$
\begin{aligned}
& \bar{n}_{A}=1+\left[\left(\mathrm{V}_{1}-\mathrm{V}_{2}\right) /\left(\mathrm{V}^{\mathrm{O}}+\mathrm{V}_{1}\right)\right]\left(\mathrm{N}^{\mathrm{O}}+\mathrm{E}^{\mathrm{O}}\right) / \mathrm{T}_{L}^{O} \\
& \bar{n}=\left[\left(\mathrm{V}_{3}-\mathrm{V}_{2}\right) /\left(\mathrm{V}^{\mathrm{O}}+\mathrm{V}_{1}\right)\right]\left[\left(\mathrm{N}^{\mathrm{O}}+\mathrm{E}^{\mathrm{O}}\right) / \mathrm{T}_{M}^{O}\right] \times 1 / \\
& {\left[\sum_{j=0}^{j} \mathrm{P}_{j}^{\mathrm{L}} H(1 / \operatorname{anti} \log \mathrm{B})\left(\mathrm{V}^{\mathrm{O}}+\mathrm{V}_{3}\right) /\left(T_{L}^{O}-\bar{n} T_{M}^{O}\right) V^{0}\right]^{\log }}
\end{aligned}
$$

The values of volumes $\mathrm{V}_{1}, \mathrm{~V}_{2} \& \mathrm{~V}_{3}$ corresponding to the same $\mathrm{pH}$ values were read from acid, ligand and complex titration curves given in graph -1 at temperature $298 \mathrm{~K}$.

\section{Proton - Ligand Stability Constant}

The ligand titration curve separates from acid titration curve at $\mathrm{pH} 5.16$ at temperature $298 \mathrm{~K}$. The ligand titration curves run parallel to the acid titration curves indicating the smooth dissociation of ligand.

The value of $\bar{n}_{A}$ at various $\mathrm{pH}$ reading [B] were calculated from the acid and ligand titration curves (Table no. 1) at temperature $298 \mathrm{~K}$.

The formation curves obtained from the plot of $\bar{n}_{A}$ vs [B] (graph NO. 2) at temperature $298 \mathrm{~K}$, show that values of $\bar{n}_{A}$ lies between 0.42 and 0.878 . This indicates that ligand is monoprotic.

Dissociation of ligand may be given as,

$$
\mathrm{LH} \rightleftarrows \mathrm{H}^{+}+\mathrm{L}^{-}
$$

Table - 1 Concentrations of solutions of metal ions, ligand, acid and salt

\begin{tabular}{ccccccc}
\hline Metal ions & $\begin{array}{c}\mathbf{V}^{\mathbf{0}} \\
\text { in } \mathbf{~} \mathbf{L}\end{array}$ & $\mathbf{Y}$ & $\mathbf{N}^{\mathbf{0}}$ & $\mathbf{E}^{\mathbf{0}}$ & $\mathbf{T}_{L}^{0}$ & $\mathbf{T}_{M}^{0}$ \\
\hline $\mathrm{Co}(\mathrm{II})$ & 100 & 1 & $1.0(\mathrm{M})$ & $1.0 \times 10^{-2}(\mathrm{M})$ & $2.5 \times 10^{-3}(\mathrm{M})$ & $5.0 \times 10^{-4}(\mathrm{M})$ \\
$\mathrm{Ni}(\mathrm{II})$ & 100 & 1 & $1.0(\mathrm{M})$ & $1.0 \times 10^{-2}(\mathrm{M})$ & $2.5 \times 10^{-3}(\mathrm{M})$ & $5.0 \times 10^{-4}(\mathrm{M})$ \\
$\mathrm{Cu}(\mathrm{II})$ & 100 & 1 & $1.0(\mathrm{M})$ & $1.0 \times 10^{-2}(\mathrm{M})$ & $2.5 \times 10^{-3}(\mathrm{M}) 5.0 \times 10^{-4}(\mathrm{M})$ \\
$\mathrm{Zn}(\mathrm{II})$ & 100 & 1 & $1.0(\mathrm{M})$ & $1.0 \times 10^{-2}(\mathrm{M})$ & $2.5 \times 10^{-3}(\mathrm{M}) 5.0 \times 10^{-4}(\mathrm{M})$ \\
\hline
\end{tabular}

The value of proton ligand stability constant was calculated by half - integral method and it was further corroborated by linear plot method ${ }^{3-4}\left(\log \bar{n}_{A} /\left(1-\bar{n}_{A}\right)\right.$ vs [B] (graph No.3) at temperature $298 \mathrm{~K}$.

Table - 2 Ligand - MNMFCI Temp. 298 $\pm 1 \mathrm{~K}$

$$
\mu^{0}=0.1(\mathrm{M}) \mathrm{KNO}_{3} \text {, Water: Dioxane 3:2(v/v) }
$$

\begin{tabular}{ccccccc}
\hline $\begin{array}{c}\text { Vol. of } \\
\text { alkali added } \\
\text { in mL }\end{array}$ & $\mathbf{H}^{+}$ & $\mathbf{H}^{+}+\mathbf{L}$ & $\begin{array}{c}\mathbf{H}^{+}+\mathbf{L}+ \\
\mathbf{C o}(\mathbf{I I})\end{array}$ & $\begin{array}{c}\mathbf{H}^{+}+\mathbf{L}+ \\
\mathbf{N i}(\mathbf{I I})\end{array}$ & $\begin{array}{c}\mathbf{H}^{+}+\mathbf{L}+ \\
\mathbf{C u}(\mathbf{I I})\end{array}$ & $\begin{array}{c}\mathbf{H}^{+}+\mathbf{L}+ \\
\mathbf{Z n}(\mathbf{I I})\end{array}$ \\
\hline 0.0 & 3.12 & 3.42 & 3.42 & 3.42 & 3.42 & 3.43 \\
0.1 & 3.22 & 3.52 & 3.42 & 3.42 & 3.42 & 3.4 \\
0.2 & 3.32 & 3.64 & 3.54 & 3.46 & 3.46 & 3.54 \\
0.3 & 3.36 & 3.74 & 3.72 & 3.72 & 3.7 & 3.7 \\
0.4 & 3.56 & 3.86 & 3.86 & 3.94 & 3.98 & 3.92 \\
0.5 & 3.88 & 4.24 & 4.34 & 4.22 & 4.22 & 4.26 \\
0.6 & 4.42 & 4.66 & 4.68 & 4.66 & 4.56 & 4.56 \\
0.7 & 5.17 & 5.27 & 5.29 & 5.28 & 5.28 & 5.3 \\
0.8 & 5.88 & 5.72 & 5.74 & 5.76 & 5.76 & 5.78 \\
0.9 & 11.6 & 7.16 & 7.47 & 6.88 & 6.86 & 7.53 \\
1.0 & 12.57 & 11.22 & 9.27 & 8.68 & 8.58 & 9.28 \\
1.1 & 12.96 & 11.82 & 10.3 & 9.56 & 9.3 & 9.76 \\
1.2 & 13.86 & 12.66 & & & & \\
\hline
\end{tabular}

The values of volumes $\mathrm{V}_{1}, \mathrm{~V}_{2} \& \mathrm{~V}_{3}$ corresponding to the same $\mathrm{pH}$ values were read from acid, ligand and complex titration curves given in graph - 6.16 page no. 249 at temperature $298 \mathrm{~K}$.

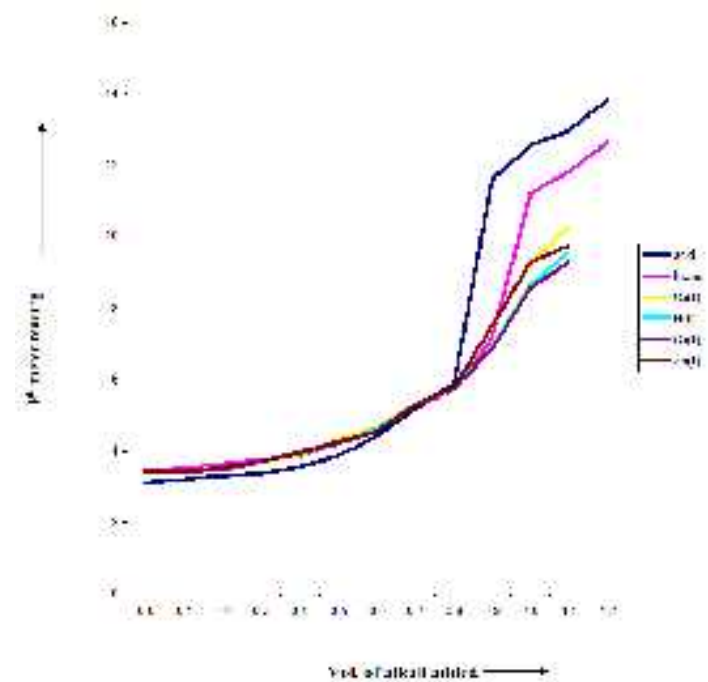

Graph No. 1 Experimental Curve with ligand MNMFCI

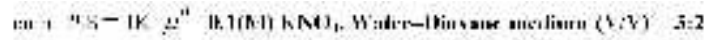

\section{Co(II) -MNMFCI System}

Complex titration curves separated from ligand mixture curve at $\mathrm{pH}=6.18$ the curves diverge at $\mathrm{pH}$ above 8.68 .

The value of $\bar{n}$ lies between 0.09 and 1.84 (table no.4, graph no $4 \mathrm{a}$ at temperature $298 \mathrm{~K}$.indicating the formation of ML and $\mathrm{ML}_{2}$ types of complexes.

From the formation curves (graph no 4) the values of $\log \mathrm{K}_{1}$ and $\log \mathrm{K}_{2}$ were calculated by half - integral method at given temperature. The values were further corroborated by mid point slope method and linear plot of $\log \bar{n} /(1-\bar{n})$ vs $\mathrm{P}^{\mathrm{L}}$ (Table no. 5 graph no. 5a) at temperature $298 \mathrm{~K}$ and plot of $\log (2-\bar{n}) /(\bar{n}-1)$ vs $\mathrm{P}^{\mathrm{L}}$.(Table no. 5 graph $\left.6 \mathrm{a}\right)$ at temperature $298 \mathrm{~K}$. 


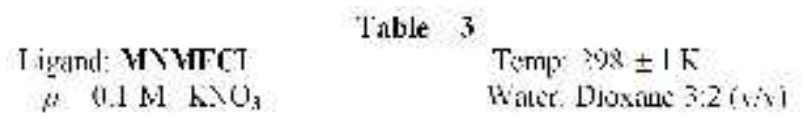

\begin{tabular}{|c|c|c|c|}
\hline [B] & $V_{2}-V_{1}$ & $\bar{n}_{A}$ & $\log \bar{n}_{A} /\left(1-\bar{n}_{A}\right)$ \\
\hline 5.0 & 0.030 & 0.878 & 0.8996 \\
\hline 5.2 & 0.032 & 0.8794 & 0.8642 \\
\hline 5.4 & 0.034 & 0.8716 & 0.8326 \\
\hline 5.6 & 0.036 & 0.8639 & 0.8020 \\
\hline 5.8 & 0.038 & 0.8560 & 0.7732 \\
\hline 6.0 & 0.040 & 0.8528 & 0.7594 \\
\hline 6.2 & 0.042 & 0.8436 & 0.7526 \\
\hline 6.4 & 0.044 & 0.8328 & 0.6940 \\
\hline 6.6 & 0.046 & 0.8189 & 0.6582 \\
\hline 6.8 & 0.049 & 0.8076 & 0.6262 \\
\hline 7.0 & 0.050 & 0.7928 & 0.5821 \\
\hline 7.2 & 0.054 & 0.7788 & 0.5483 \\
\hline 7.4 & 0.056 & 0.7682 & 0.5190 \\
\hline 7.6 & 0.060 & 0.7528 & 0.4822 \\
\hline 7.8 & 0.064 & 0.7366 & 0.4374 \\
\hline 8.0 & 0.068 & 0.7242 & 0.4178 \\
\hline 8.2 & 0.072 & 0.7156 & 0.4019 \\
\hline 8.4 & 0.076 & 0.7037 & 0.3746 \\
\hline 8.6 & 0.078 & 0.6928 & 0.3528 \\
\hline 8.8 & 0.080 & 0.6756 & 0.3179 \\
\hline 9.0 & 0.084 & 0.6592 & 0.2882 \\
\hline 9.2 & 0.808 & 0.6394 & 0.2486 \\
\hline 9.4 & 0.094 & 0.6192 & 0.2122 \\
\hline 9.6 & 0.101 & 0.5993 & 0.1744 \\
\hline 9.8 & 0.102 & 0.5884 & 0.1538 \\
\hline 10.0 & 0.106 & 0.5682 & 0.1178 \\
\hline 10.2 & 0.110 & 0.5526 & 0.0888 \\
\hline 10.4 & 0.116 & 0.5266 & 0.0478 \\
\hline 10.6 & 0.124 & 0.4984 & 0.0026 \\
\hline 10.8 & 0.132 & 0.4675 & -0.0558 \\
\hline 11.0 & 0.144 & 0.4210 & -0.1422 \\
\hline
\end{tabular}

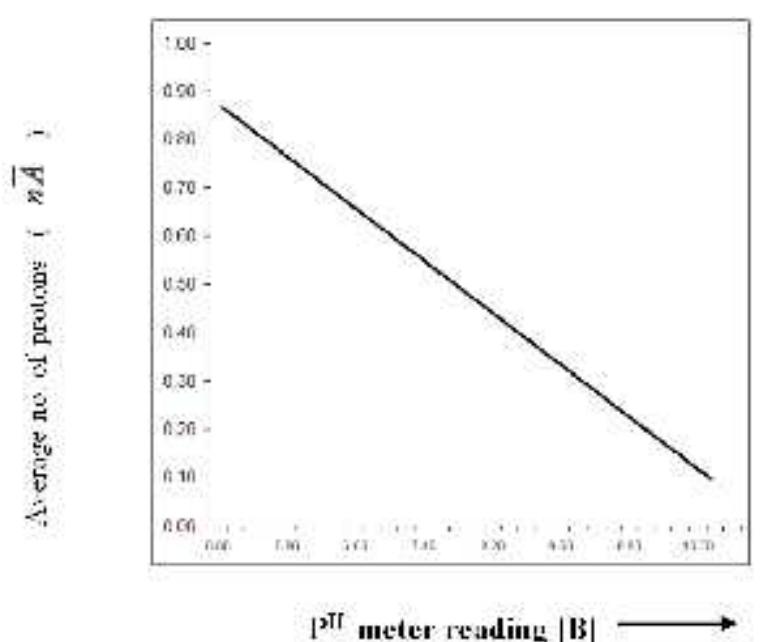

Graph No. 2 Formation Curve of ligand MNMFCI

Plot of $\bar{n}_{A}$ Vs [B] $\mu=0.1 \mathrm{M} \mathrm{KNO}_{3}$ Water: Dioxane 3:2(v/v)

\section{Ni(II) - MNMFCI System}

The complex titration curves crossed the ligand titration curve at $\mathrm{pH}=5.16$ indicating the start of complexation. The curve increases regularly upto $\mathrm{pH}=7.78$ indicating quick but incomplete dissociation of ligand.

No turbidity appears, hence hydrolysis does not take place values of $\bar{n}$ falls between 0.45 and 1.972 (graph 4b, Table no. 6.) at temperature $298 \mathrm{~K}$ indicating the formation of $\mathrm{ML}$ and $\mathrm{ML}_{2}$ type of complexes.

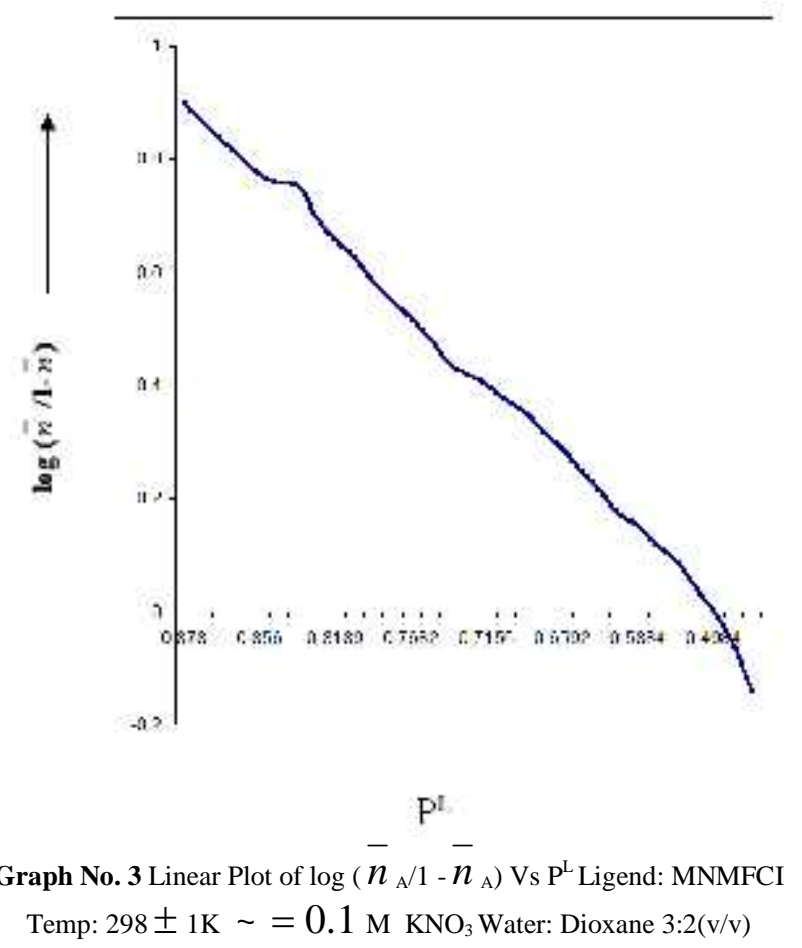

From the formation curves, (graph $4 b$ ) the values of $\log \mathrm{K}_{1}$ and $\log \mathrm{K}_{2}$ were calculated by half integral method at given temperature and verified by mid - point slope method and linear plot of $\log (n / 1-\bar{n})$ vs $\mathrm{p}^{\mathrm{L}}$ ( graph $5 \mathrm{~b}$, table no. 7 at temperature $298 \mathrm{~K}$ and liner plot of $\log (2-n / n-1) \mathrm{vs}^{\mathrm{L}}$ (graph no. 6b table no. 7) at temperature $298 \mathrm{~K}$.

\section{Cu(II) - MNMFCI System}

The complex, titration curve separated from ligand mixture curve at $\mathrm{pH} 5.28$ indicating the start of complex formation.

As the metal titration curves did not join up and run parallel to the ligand titration curves indicating liberation of extra proton due to hydrolysis. Precipitation was observed at $\mathrm{pH}=8.36$. Hence, in order to preclude error due to hydrolysis in the calculation of $\bar{n}$, only the lower $\mathrm{pH}$ region of titration curves were used.

The values of $\bar{n}$ lies between 0.04 to 1.90 graph -3 table no. 8 at temperature $298 \mathrm{~K}$ indicating the formation of $\mathrm{ML}$ and $\mathrm{ML}_{2}$ type of complexes.

From the formation curves (graph $-4 \mathrm{c}$ and table no. 8) of $\bar{n}$ vs $\mathrm{P}^{\mathrm{L}}$, the value of $\log \mathrm{K}_{1}$ and $\log \mathrm{K}_{2}$ at the given two temperatures were calculated by half - integral method. It was verified by the mid - point slope method and linear plot of log $\bar{n} /(1-\bar{n})$ vs $\mathrm{P}^{\mathrm{L}}$ graph no. 5c p, table no. 9 and plot of $\log (2$ $-\bar{n}) /(\bar{n}-1)$ vs $\mathrm{P}^{\mathrm{L}}$ graph no.6c and table no. 9 at temperature $298 \mathrm{~K}$.

\section{Zn(II) - MNMFCI System}

The complex curves separated from ligand titration curves at $\mathrm{pH}=5.89$ and diverges at $\mathrm{pH}$ above 8.65.

During the titration no turbidity appears, hence hydrolysis does not take place. 
The values of $\bar{n}$ lies between 0.04 to 1.81 (Table - 10, graph $4 \mathrm{~d}$ at temperature $298 \mathrm{~K}$ indicating the formation of $\mathrm{ML}$ and $\mathrm{ML}_{2}$ type of complexes.

From the formation curve, (graph - 4d) at $298 \mathrm{~K}$ values of $\log$ $\mathrm{K}_{1}$ and $\log \mathrm{K}_{2}$ were calculated by half - integral method.

It was further verified by mid-point slope method and linear plot of $\log \bar{n} /(1-\bar{n})$ vs $\mathrm{P}^{\mathrm{L}}$ graph no. 5d table no. 11 at temperature $298 \mathrm{~K}$ and plot of $\log (2-\bar{n}) /(\bar{n}-1)$ vs $\mathrm{P}^{\mathrm{L}}$ (graph no.-6d, Table no.11) at temperature $298 \mathrm{~K}$.

Table 4 Co (II) + MNMFCI Temp: $298 \pm 1 \mathrm{~K}$ $\mu=0.1 \mathrm{M} \mathrm{KNO}_{3}$ Water: Dioxane 3:2(v/v)

\begin{tabular}{cccc}
\hline$[\mathbf{B}]$ & $\mathbf{V}_{\mathbf{3}}-\mathbf{V}_{\mathbf{2}}$ & $\bar{n}$ & $\mathbf{P}^{\mathbf{L}}$ \\
\hline 6.0 & 0.006 & 0.0936 & 7.2140 \\
6.2 & 0.008 & 0.1898 & 7.0226 \\
6.4 & 0.010 & 0.2886 & 6.8318 \\
6.6 & 0.015 & 0.4150 & 6.6396 \\
6.8 & 0.020 & 0.5210 & 6.4540 \\
7.0 & 0.024 & 0.6320 & 6.2646 \\
7.2 & 0.032 & 0.7962 & 6.0820 \\
7.4 & 0.034 & 0.9386 & 5.8964 \\
7.6 & 0.042 & 1.1452 & 5.7192 \\
7.8 & 0.046 & 1.3078 & 5.5374 \\
8.0 & 0.054 & 1.5220 & 5.3636 \\
8.2 & 0.065 & 1.8464 & 5.2080 \\
\hline
\end{tabular}

Table - 5 Co(II) + MNMFCI Temp: $298 \pm 1 \mathrm{~K}$ $\mu=0.1 \mathrm{M} \mathrm{KNO}_{3}$ Water: Dioxane 3:2(v/v)

\begin{tabular}{|c|c|c|c|}
\hline$\frac{\log }{n} /(1-\bar{n})$ & $\mathbf{P}^{\mathrm{L}}$ & $\begin{array}{l}\log (2- \\
\left.-\frac{-}{n}\right) /(n-1)\end{array}$ & $\mathbf{P}^{\mathbf{L}}$ \\
\hline-0.8494 & 7.0226 & 0.7696 & 5.7192 \\
\hline-0.3910 & 6.8318 & 0.1978 & 5.5476 \\
\hline-0.1486 & 6.6396 & -0.1846 & 5.3750 \\
\hline 0.2350 & 6.2648 & -0.7409 & 5.2078 \\
\hline 0.5912 & 6.0814 & & \\
\hline
\end{tabular}

Table - 6 Ni (II) + MNMFCI Temp: $298 \pm 1 \mathrm{~K}$ $\mu=0.1 \mathrm{M} \mathrm{KNO}_{3}$ Water: Dioxane 3:2(v/v)

\begin{tabular}{cccc}
\hline $\mathbf{B}$ & $\mathbf{V}_{\mathbf{3}}-\mathbf{V}_{\mathbf{2}}$ & $\bar{n}$ & $\mathbf{P}^{\mathbf{L}}$ \\
\hline 5.2 & 0.003 & 0.0454 & 8.0096 \\
5.4 & 0.004 & 0.1376 & 7.8178 \\
5.6 & 0.008 & 0.2318 & 7.6266 \\
5.8 & 0.012 & 0.3512 & 7.4374 \\
6.0 & 0.020 & 0.4935 & 7.2476 \\
6.2 & 0.024 & 0.5932 & 7.0618 \\
6.4 & 0.028 & 0.6980 & 6.8716 \\
6.6 & 0.036 & 0.8552 & 6.6868 \\
6.8 & 0.040 & 1.0413 & 6.5070 \\
7.0 & 0.048 & 1.2390 & 6.3732 \\
7.2 & 0.056 & 1.4634 & 6.1568 \\
7.4 & 0.064 & 1.6948 & 5.9864 \\
7.6 & 0.070 & 1.9720 & 5.8244 \\
\hline
\end{tabular}

Table - $7 \mathrm{Ni}(\mathrm{II})+$ MNMFCI Temp: $298 \pm 1 \mathrm{~K}$ $\mu=0.1 \mathrm{M} \mathrm{KNO}_{3}$ Water: Dioxane 3:2(v/v)

\begin{tabular}{|c|c|c|c|}
\hline Log & \multicolumn{3}{|c|}{$\log (2-$} \\
\hline $\bar{n} /(1-\bar{n})$ & $\mathbf{P}^{\mathbf{L}}$ & $\bar{n}) /(\bar{n}-1)$ & $\mathbf{P}^{\mathrm{L}}$ \\
\hline-0.7956 & 7.8182 & 0.5026 & 6.3232 \\
\hline-0.5202 & 7.6264 & 0.0632 & 6.1518 \\
\hline-0.2668 & 7.4378 & -0.3596 & 5.9860 \\
\hline 0.1640 & 7.0668 & & \\
\hline 0.3642 & 6.8712 & & \\
\hline 0.7704 & 6.6816 & & \\
\hline
\end{tabular}

Table - $8 \mathrm{Cu}(\mathrm{II})+$ MNMFCI Temp: $298 \pm 1 \mathrm{~K}$ $\mu=0.1 \mathrm{M} \mathrm{KNO}_{3}$ Water: Dioxane 3:2(v/v)

\begin{tabular}{cccc}
\hline $\mathbf{B}$ & $\mathbf{V}_{\mathbf{3}}-\mathbf{V}_{\mathbf{2}}$ & $\bar{n}$ & $\mathbf{P}^{\mathbf{L}}$ \\
\hline 5.0 & 0.004 & 0.0451 & 8.2098 \\
5.2 & 0.006 & 0.0912 & 8.0140 \\
5.4 & 0.008 & 0.1606 & 7.8212 \\
5.6 & 0.012 & 0.2316 & 7.5658 \\
5.8 & 0.014 & 0.3742 & 7.3386 \\
6.0 & 0.020 & 0.5174 & 7.2540 \\
6.2 & 0.028 & 0.6410 & 7.0652 \\
6.4 & 0.034 & 0.7946 & 6.8820 \\
6.6 & 0.042 & 0.9772 & 6.7015 \\
6.8 & 0.048 & 1.1410 & 6.5178 \\
7.0 & 0.052 & 1.3662 & 6.3438 \\
7.2 & 0.064 & 1.6178 & 6.1762 \\
7.4 & 0.078 & 1.9040 & 6.1762 \\
\hline
\end{tabular}

Table - $9 \mathrm{Cu}(\mathrm{II})+\mathrm{MNMFCI}$ Temp: $298 \pm 1 \mathrm{~K}$ $\mu=0.1 \mathrm{M} \mathrm{KNO}_{3}$ Water: Dioxane 3:2(v/v)

\begin{tabular}{|c|c|c|c|}
\hline Log & \multicolumn{3}{|c|}{$\log (2-$} \\
\hline $\bar{n} /(1-\bar{n})$ & $\mathbf{P}^{\mathrm{L}}$ & $\bar{n}) /(\bar{n}-1)$ & $\mathbf{P}^{\mathrm{L}}$ \\
\hline-0.7174 & 7.8202 & 0.7874 & 6.5188 \\
\hline-0.5202 & 7.6266 & 0.2396 & 6.3446 \\
\hline-0.2228 & 7.4396 & -0.2082 & 6.1762 \\
\hline 0.2512 & 7.0654 & & \\
\hline 0.5874 & 6.8816 & & \\
\hline
\end{tabular}

Table $10 \mathrm{Zn}(\mathrm{II})+$ MNMFCI Temp:298 $\pm 1 \mathrm{~K}$ $\mu=0.1 \mathrm{M} \mathrm{KNO}_{3}$ Water: Dioxane 3:2(v/v)

\begin{tabular}{cccc}
\hline$[\mathbf{B}]$ & $\mathbf{V}_{\mathbf{3}}-\mathbf{V}_{\mathbf{2}}$ & $\bar{n}$ & $\mathbf{P}^{\mathbf{L}}$ \\
\hline 6.0 & 0.004 & 0.0472 & 7.2102 \\
6.2 & 0.006 & 0.0948 & 7.0166 \\
6.4 & 0.008 & 0.1446 & 6.8188 \\
6.6 & 0.010 & 0.2194 & 6.6254 \\
6.8 & 0.012 & 0.3222 & 6.4352 \\
7.0 & 0.016 & 0.4554 & 6.2478 \\
7.2 & 0.022 & 0.5906 & 6.0604 \\
7.4 & 0.028 & 0.7802 & 6.8798 \\
7.6 & 0.036 & 0.9856 & 5.7014 \\
7.8 & 0.044 & 1.2518 & 5.5313 \\
8.0 & 0.052 & 1.5214 & 5.3645 \\
8.2 & 0.066 & 1.8180 & 5.2036 \\
\hline
\end{tabular}

Table - $11 \mathrm{Zn}(\mathrm{II})+$ MNMFCI Temp: $298 \pm 1 \mathrm{~K}$ $\mu=0.1 \mathrm{M} \mathrm{KNO}_{3}$ Water: Dioxane 3:2(v/v)

\begin{tabular}{cccc}
\hline $\log \bar{n} /(1-\bar{n})$ & $\mathbf{P}^{\mathrm{L}}$ & $\bar{n}) /(\bar{n}-1)$ & $\mathbf{P}^{\mathbf{L}}$ \\
\hline-0.5502 & 6.6254 & 0.4728 & 5.5314 \\
-0.3228 & 6.4352 & 0.0376 & 5.3642 \\
-0.0782 & 6.2474 & -0.6530 & 5.2031 \\
0.1586 & 6.0606 & -0.7228 & 5.0321 \\
0.5554 & 5.8798 & -0.8315 & 4.9832 \\
& & -0.9232 & 4.8215 \\
\hline
\end{tabular}




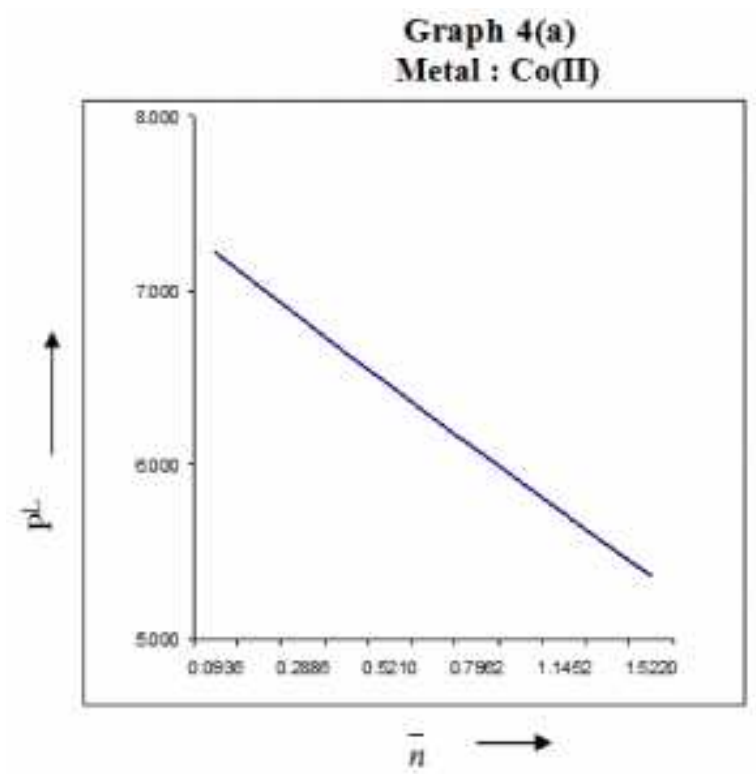

Graph 4 (c)

Metal : Cu(II)

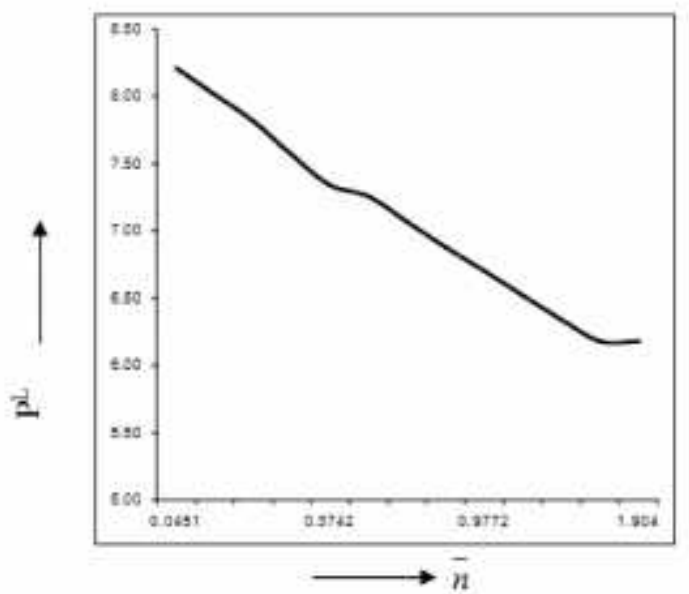

Graph 4 (b)

Metal : Ni(II)

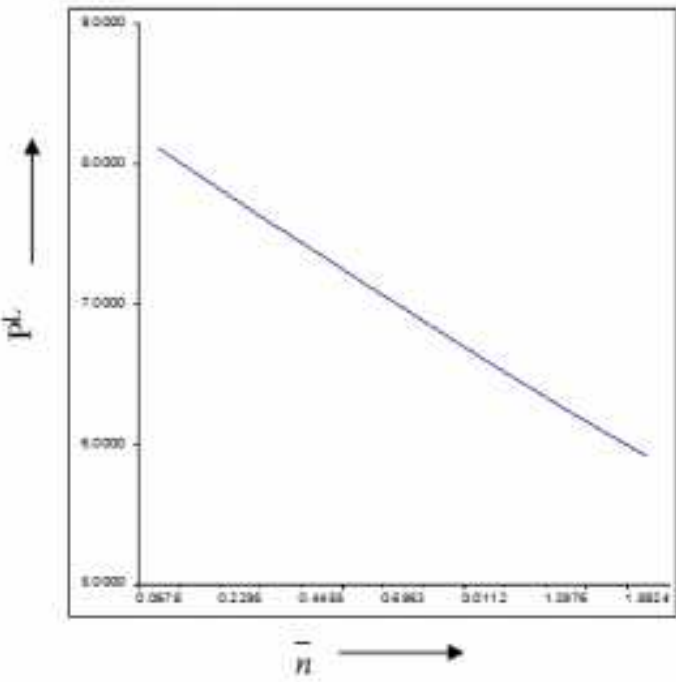

Graph 4 (d)

Metal : Zn(II)

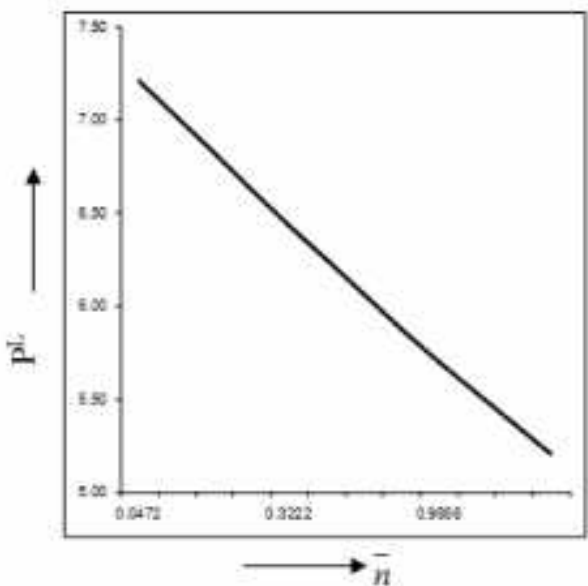

Graph No. 4 Formation curve Plot of $\bar{n}$ Vs $\mathrm{P}^{\mathrm{L}}$ Ligand: MNMFCI Temp:298 $\pm 1 \mathrm{~K} \mu=0.1 \mathrm{M} \mathrm{KNO}_{3}$ Water: Dioxane 3:2(v/v)

Givaph $5(a)$

Metal : Co\{II

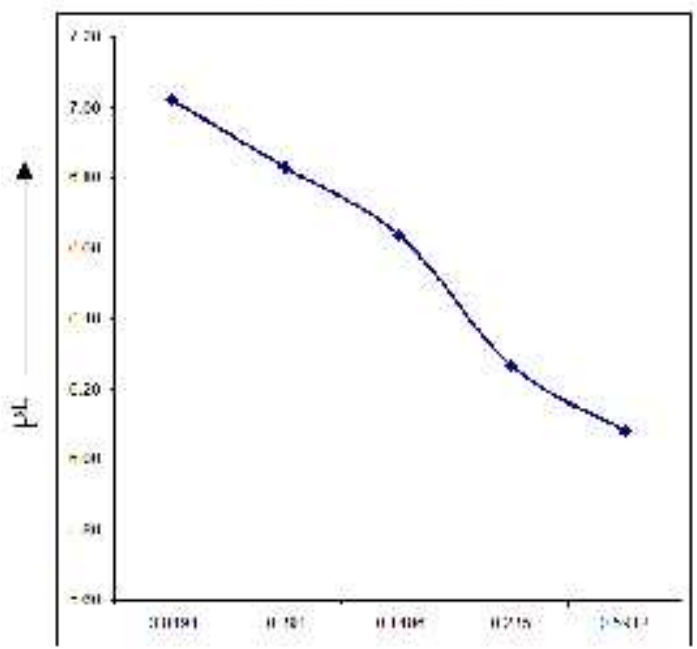

$\log (n ; 1-n)$
Graph $5(\mathrm{~b})$

Meta : $\mathrm{Ni}(\boldsymbol{H})$

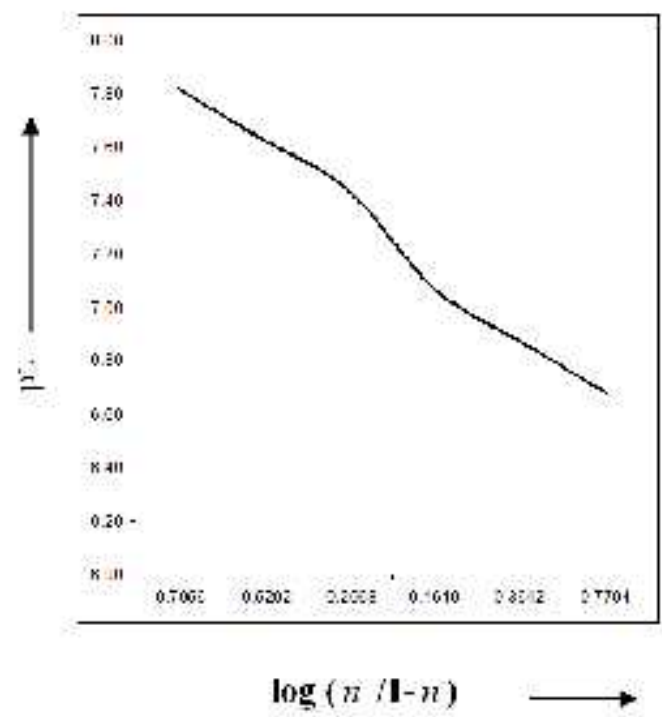




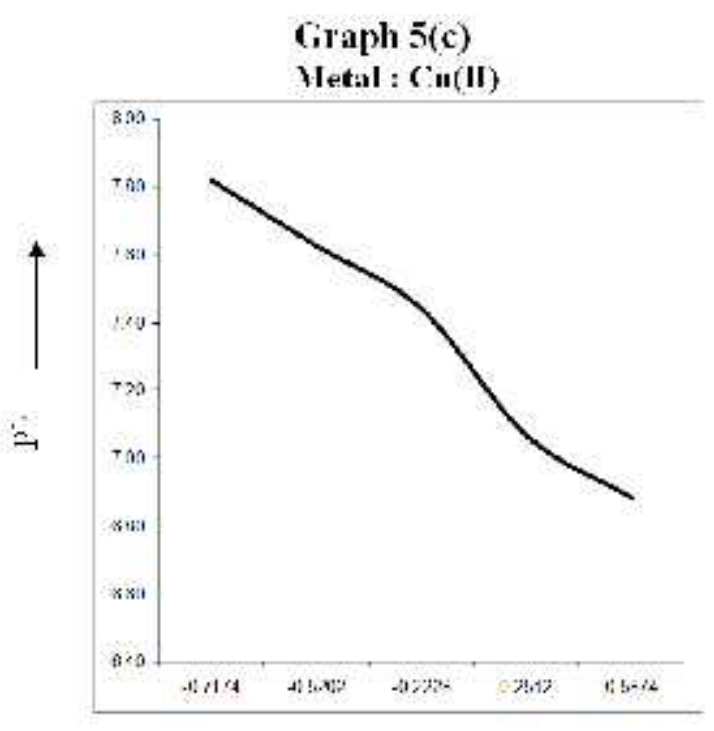

$\operatorname{lng}(n / 1 n)$

Graph No. 5 Linear plot of $\log (\bar{n} / 1-\bar{n})$ Vs $\mathrm{P}^{\mathrm{L}}$ Ligand : MNMFCI Temp

Graph 6(a)

Metal : Co(II)

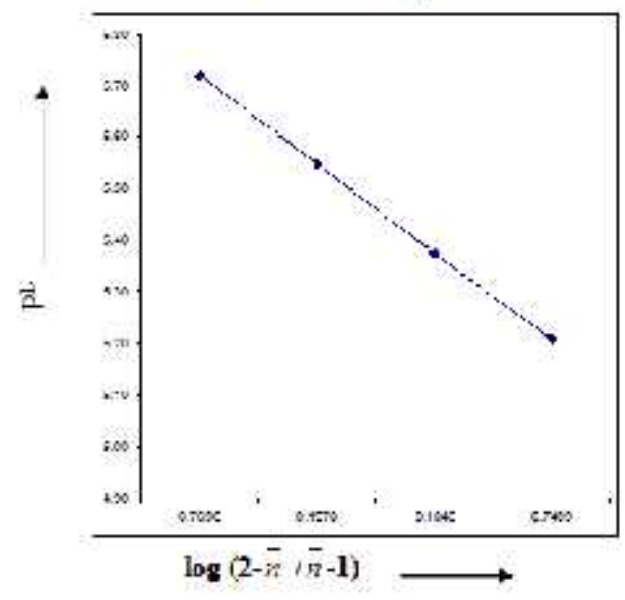

Graph $6(c)$

Metal : Cu(II)

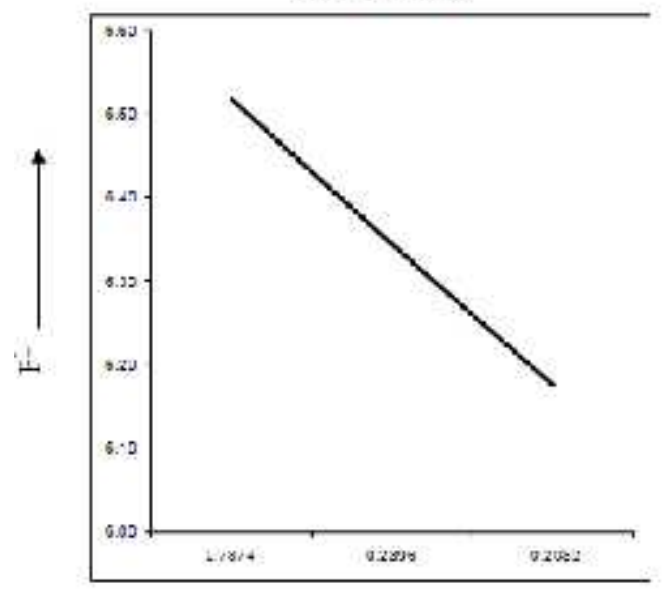

$\log (2-\bar{n}, \bar{n}-1)$
Graph 5 (d)

Nletal : Y an (II)

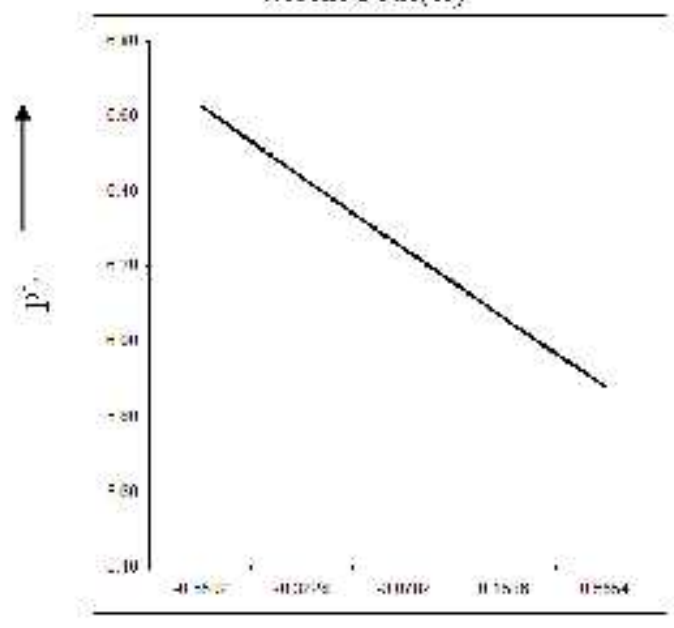

$\log (f / 1 \quad s)$

$298 \pm 1 \mathrm{~K} \mu=0.1 \mathrm{M} \mathrm{KNO}_{3}$ Water: Dioxane 3:2(v/v) Graph $\sigma(b)$ Metal: Ni(II)

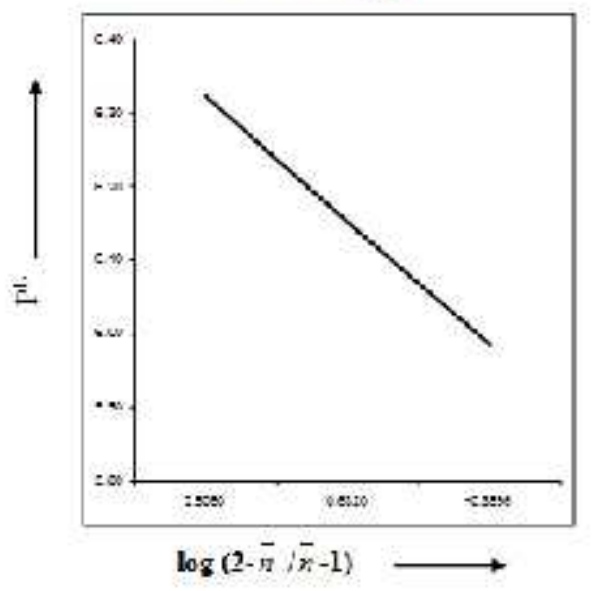

Gripli 6 (i1) Metal : Zn(II)

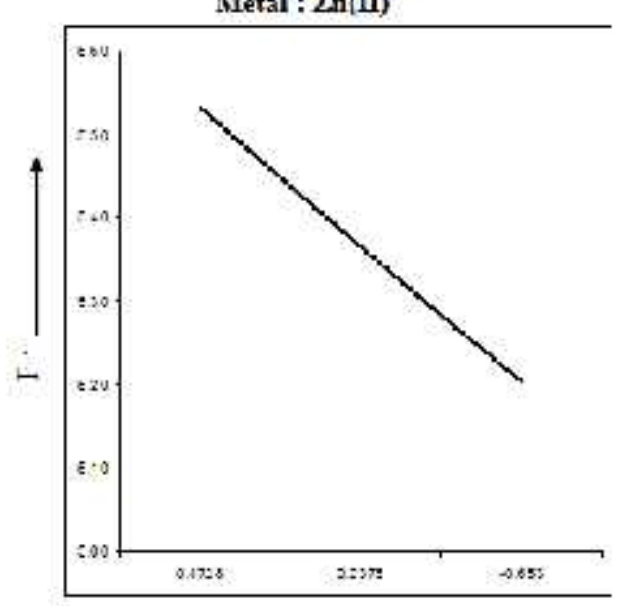

$\log (2-\bar{n} / \bar{n}-1)$

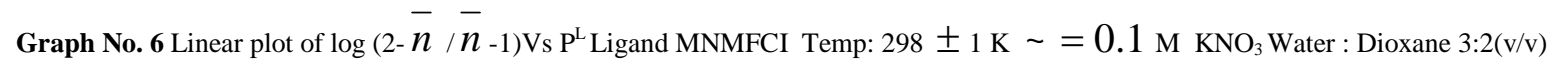


The value of protonation constant and stepwise stability constant obtained from different computational methods at temperatures $298 \mathrm{~K}$ are summarized in table no. 12. The different computational methods are :-

1. Half - integral method

2. Mid - point slope calculation method and

3. Straight - line plot method.

Table no 12 Values of protonation constant of ligand and stepwise stability constant of complexes of $\mathrm{Co}$ (II),

$\mathrm{Ni}(\mathrm{II}), \mathrm{Cu}(\mathrm{II}) \& \mathrm{Zn}(\mathrm{II})$ with ligand (MNMFCI at temperatures $298 \mathrm{~K}$.

\begin{tabular}{|c|c|c|}
\hline \multirow[t]{2}{*}{ Metal ions } & \multicolumn{2}{|c|}{$\begin{array}{c}\text { Ligand } \\
\text { MNMFCI }\end{array}$} \\
\hline & $\log K_{1}$ & $\log K_{2}$ \\
\hline & 11.20 & - \\
\hline & - & - \\
\hline MNMFCI & 11.22 & - \\
\hline \multirow[t]{3}{*}{$\mathrm{Co}(\mathrm{II})$} & 7.18 & 6.18 \\
\hline & 7.22 & 6.20 \\
\hline & 7.18 & 6.10 \\
\hline \multirow[t]{3}{*}{$\mathrm{Ni}(\mathrm{II})$} & 7.06 & 5.95 \\
\hline & 7.18 & 6.24 \\
\hline & 6.44 & 5.36 \\
\hline \multirow[t]{3}{*}{$\mathrm{Cu}(\mathrm{II})$} & 6.30 & 5.36 \\
\hline & 6.46 & 5.46 \\
\hline & 6.16 & 5.42 \\
\hline \multirow[t]{3}{*}{$\mathrm{Zn}(\mathrm{II})$} & 6.04 & 5.38 \\
\hline & 6.12 & 5.36 \\
\hline & 6.14 & 5.32 \\
\hline
\end{tabular}

The most representative values of $\log \mathrm{K}_{1}$ and $\log \mathrm{K}_{2}$ are given in table no. 13 .

Table - $\mathbf{1 3} \log$ of stepwise and overall stability constants of complex compounds of various metals with ligand

MNMFCI at $298 \mathrm{~K} . \mu^{0}=0.10(\mathrm{M}) \mathrm{KNO}_{3}$ Water dioxane medium $(\mathrm{V} / \mathrm{V})=3: 2$

\begin{tabular}{cccc}
\hline & \multicolumn{3}{c}{ Ligand (MNMFCI) } \\
\cline { 2 - 4 } System & $\log \mathbf{K}_{\mathbf{1}}$ & $\log \mathbf{K}_{\mathbf{2}}$ & $\log \beta$ \\
\hline HNMFCI & 11.88 & & 11.88 \\
MNMFCI & 7.46 & 7.42 & 14.88 \\
Cu (II) & 7.34 & 7.26 & 14.60 \\
Ni (II) & 6.75 & 6.68 & 13.43 \\
Co (II) & 6.58 & 6.42 & 13.0 \\
Zn(II) & &
\end{tabular}

MNMFCI -Cu(II) > Ni (II) > Co (II) $>\mathrm{Zn}$ (II)

\section{Discussion}

The values of stepwise stability constants and over all stability constants are given in Table No. 13. For the given ligand the stability constants of complexes for different metals show the sequence

$\mathrm{Cu}(\mathrm{II})>\mathrm{Ni}(\mathrm{II})>\mathrm{Co}(\mathrm{II})>\mathrm{Zn}(\mathrm{II})$

This is natural order given by Irving - William ${ }^{15}$. A theoretical justification of the order of stability constants follows from the consideration of the reciprocal of the ionic radii and $2^{\text {nd }}$ ionization enthalpy of metal. Calvin - Bjerrum titration technique modified by Irving and Rossotti was used to determine the practical proton ligand and metal ligand stability constants at constant ionic strength maintained by using dilute $\mathrm{KNO}_{3}$ solution. The advantage of Irving and Rossotti method is that the formation constant of metal chelates can be obtained without converting the $\mathrm{pH}-$ meter reading [B] to stoichiometric hydrogen ion concentration and without knowing the stiochiometric concentration of neutral salts added to maintain ionic strength. This method is valid for both aqueous and non-aqueous medium.

The nitrate $\left(\mathrm{NO}_{3}^{-}\right)$ion has very slight complexing tendency. Therefore, competition between nitrate ion and the ligand under study is of no importance ${ }^{16}$.

The stability of the chelates is greatly affected by the electron density around the imino nitrogen $(-\mathrm{C}=\mathrm{N}-)^{17}$. Higher the electron density around the nitrogen atom, stronger is the metal ligand bond.

The difference between the successive stepwise stability constant is large, which suggest that the formation of ML and $\mathrm{ML}_{2}$ chelates take place. The results obtained are in conformity of our previous studies and the study of other workers ${ }^{17-24}$

\section{References}

1. Irving H.M., Rossotti H.S.,: J. Chem Soc., 2904, 1954.

2. Bjerrum, J: Metal amine formation in aqueous solution. P. Hasse and Sons. Copenhagen 1941.

3. Liu C. M.; Xiong R.G.; You X. Z.; Liu Y.J.; Cheung K.K., Polyhedron, 15 1996, 45651.

4. Djebbar S.S.; Benali B.O.; Deloume J.P., Transit Metal Chem., 23, 1998,443. \&. Polyhedron, 16, 1997, 2175.

5. Hamada Y.J., IEEE Trans. Electron Devices, 44, 1997, 1208.

6. Srivastava R.S., Ind. J. Chem., 29, 1990, 1024 - 1026.

7. Waish C., Nature, 409, 2001, 226.

8. He L.; Gou S. H.; Shi Q.F., J. Chem. Crystallogr., 29, 1999, 207.

9. Wu J.C.; Tang N.: Liu W.S.; Tan M.Y. ; Chan A.S., Chin Chem. Lett., 12, 2001, 757.

10. Pandey V. K.; Sarah, T and Zehra T: Indian J. Chem. 43 B, 2004, 180

11. Joshi, L.D.; Kumar, R. and Parmar, S.S., Curr.Sci. $42,1973,847$

12. Otto, H. and Houlohan, W.W.; Swiss Pat 1971, 499, 540, Chem Abstr. 75, 1771,20435

13. Irving, H. and Rossotti, H. J.: J. Chem. Soc. 3397 (1953)

14. Bhattacharrya P.; Parr J.; Ross A. T., Chem. Soc. Dalton, 1998, 3149.

15. Brown D. H., Smith W.E., Teape J.W. et al., J. Med. Chem., 23(7), 1980,729.

16. Mukherjee, S. K. and Rawat N.S., J. Ind. Chem. Soc. $58,614,1981$

17. P. M. Mishra et al: Asian Journal of Chemistry Vol. 21(7) p - 5055 - 5060 (2009). \& Vol. 21(9) p -7195 7199(2009).

18. P. M. Mishra et al: Journal of Ultra Chemistry Vol. 5(2) p - 131-136 (2009). \& Vol. 9(1) p - 149 - 155 (2013).\& Vol. 12 No.-2, p. 38-51 (2016

19. P. M. Mishra et al: Rasayan Journal of Chemistry Vol. 4(2) p-303-319 (2011).

20. T. Suresh et. al: J. Ind. Council of Chem. Vol. 24(1) pp - 123 (2007) \&. Vol. 25(2) pp - 68-70 (2008)

21. P. M. Mishra, Bimlesh Kumar Yadav: Ultra Scientist Vol. 27(3) B, p - 139-154 (2015)

22. P. M. Mishra: Journal of Applicable Chemistry Vol. 5 No.-5, p. 1163-74 (2016) 
23. P. M. Mishra: Oriental Journal of Chemistry Vol. 32 No.-5, (2016)
24. P. M. Mishra et al. International Journal of Current Advanced Research Vol 5, Issue 12, pp 1606-1611, (2016)

\section{How to cite this article:}

Prem Mohan Mishra et al (2017) ' Determination Of Thermodynamic Stability Constant Of Bivalent Transition metal complexes with schiffs base as ligands', International Journal of Current Advanced Research, 06(03), pp. $2480-2487$. DOI: http://dx.doi.org/10.24327/ijcar.2017.2487.0033 